\title{
Caracterización funcional de la RAP Eje Cafetero, Colombia
}

\author{
Caracterização funcional do RAP Eje Cafetero, Colombia \\ Functional characterization of the RAP Eje Cafetero, Colombia
}

Mario Alberto Gaviria Ríos[a] [D, Andrés Felipe Aristizábal Toro[b] (D)

[a] Universidad Católica de Pereira, Pereira, Risaralda, Colombia

[b] Universidad del Quindío, Armenia, Quindío, Colombia

Cómo citar: Gaviria Ríos, M. A., \& Aristizábal Toro, A. F. (2020). Caracterización funcional de la RAP Eje Cafetero, Colombia. urbe. Revista Brasileira de Gestão Urbana, 12, e20200052. https://doi.org/10.1590/2175-3369.012.e20200052

\section{Resumen}

Se presenta una caracterización funcional de la región del centro occidente colombiano, RAP Eje cafetero, en la que se identifican sus centros y subcentros regionales. A nivel de resultados, se observó en ella una organización de carácter policéntrico en la que tres de sus nodos centrales (Pereira, Manizales, Armenia) se desplegaron a partir de un proceso paralelo de expansión industrial y de producción cafetera, y aprovechan ventajas de conectividad y proximidades geográficas y culturales; en tanto el nodo restante (La Dorada) se apalanca en su localización estratégica, que le ofrece ventajas para la conectividad con la costa Atlántica y el centro del país, y atiende a una población que por distancia está fuera del área de influencia del resto de centros regionales.

Palabras clave: Funciones urbanas. Policentrismo. Región. Geografía económica. Economía urbana.

\section{Resumo}

É apresentada uma caracterização das funções da região centro-oeste da Colombia, RAP Eje cafetero, identificando seus centros e subcentros regionais. No nível dos resultados, observou-se uma organização policêntrica na qual três de seus nós centrais (Pereira, Manizales, Armenia) eles se desenrolaram por meio de um processo paralelo de expansão industrial e produção de café, e aproveitar as vantagens da conectividade e proximidade geográfica e cultural; enquanto o nó restante (La Dorada) é alavancado em sua localização estratégica, que oferece vantagens para a conectividade com a costa atlântica e o centro do país, e atende a uma população que, à distância, está fora da área de influência do restante centros regionais.

Palavras-chave: Funções urbanas. Policentrismo. Região. Geografia econômica. Economia urbana.

\section{Abstract}

It is presented a functional characterization of the western central Colombian region, RAP Eje cafetero, where its regional centers and sub-centers are identified. At the results level, a polycentric organization was observed in which three of its central nodes (Pereira, Manizales, Armenia) were deployed from a 
parallel process of industrial expansion and coffee production, and take advantage of connectivity and geographical and cultural proximity. While the remaining node (La Dorada) is leveraged in its strategic location, which offers advantages for connectivity with the Atlantic coast and the center of the country, and serves a population that by distance is outside the area of influence of the other Regional centers.

Keywords: Urban functions. Polycentrism. Region. Economic geography. Urban economy.

\section{Introducción}

Colombia es un Estado unitario descentralizado declarado por la Constitución de 1991, dónde se dejó por sentado que además de la división general del territorio, que asume como niveles los departamentos y municipios, podrían considerarse otros fraccionamientos territoriales que determine la ley y se consideren necesarios para el cumplimiento de las funciones y servicios a cargo del Estado.

Las orientaciones de la Constitución Política para favorecer una nueva regionalización en Colombia están especialmente contenidas en sus Artículos 306, que permite a los departamentos constituirse en Regiones Administrativas y de Planificación (RAP), y 307, que construye los cimientos de lo que se considera "[...] un estadio de desarrollo superior en su vida administrativa mediante la figura de la constitución de Regiones Entidad Territorial (Colombia, 1991)", para las cuales el Gobierno Nacional puede asignar recursos del presupuesto general de la Nación.

Los Consejos Regionales de Política Económica y Social (CORPES), creados mediante la ley 76 de 1985 como una iniciativa para la planificación del desarrollo económico y social, constituyen un antecedente inmediato de las regiones de planificación. Pero es a partir de la expedición de la Constitución de 1991 que se desarrolla el marco institucional de las RAP como esquemas asociativos de entidades territoriales, que según el Departamento Nacional de Planeación (DNP) surgen como instrumentos de articulación y desarrollo territorial para abordar problemas y temáticas que, por su naturaleza y dimensión, no pueden ser atendidas en forma efectiva de manera individual (Colombia, 2013).

Con el fin de reglamentar la creación de nuevos entes territoriales y ejecutar planes conforme a objetivos específicos en términos de organización territorial, se expidió la Ley Orgánica de Ordenamiento Territorial (LOOT) o Ley 1454 de 2011, que define los distintos esquemas asociativos de entidades territoriales y desarrolla un concepto de regionalización animado por propósitos de planificación; promoviéndose la conformación de regiones como marco de relaciones geográficas, económicas, culturales y funcionales, que sirvan a la creación de entes territoriales que generen valor para sus habitantes y la Nación colombiana.

A partir de la promulgación de la LOOT en Colombia se han conformado las RAP Caribe, Pacifico, Eje cafetero y la RAPE${ }^{1}$ Región Central. La RAP Eje Cafetero, aprobada por la Comisión de Ordenamiento Territorial del Senado de la República en mayo de 2018, está integrada por los departamentos de Caldas, Quindío y Risaralda, fue concebida con el objetivo de promover el desarrollo económico y social de la región Eje cafetero, y marca un hito en los propósitos de integración y fortalecimiento de las relaciones regionales con miras a consolidar proyectos estratégicos de impacto regional.

En este contexto de integración regional, se consideró fundamental avanzar en estudios que sirvan a la caracterización de la RAP Eje cafetero, explorando en ella las centralidades, a partir de las morfologías y funcionalidades de sus municipios, las jerarquías urbanas y las condiciones de distribución de su población, como elementos que inciden en la cohesión territorial y sirven a las estrategias de ordenamiento del territorio en proceso de configuración.

Este artículo da cuenta de la caracterización funcional de la RAP. Inicialmente se revisan las experiencias de regionalización en Colombia, que anteceden los procesos de asociatividad territorial

\footnotetext{
${ }^{1}$ Región Administrativa y de Planificación Especial (RAPE), que de acuerdo con la LOOT se conforma por la asociación entre entidades territoriales departamentales y el Distrito Capital.
} 
propuestos en la Ley Orgánica de Ordenamiento Territorial (LOOT) y dan origen a la RAP Eje cafetero. Posterior a ello, se identifican los centros y subcentros regionales en la RAP, desde un enfoque funcional.

\section{Los procesos de regionalización en Colombia}

Las ciencias sociales reconocen la relevancia de la región dado su papel como marco referencial de análisis, planificación y gestión del desarrollo a partir de políticas públicas de abajo - arriba. Como lo describen Mateo-Rodríguez \& Bollo-Manent (2016), la región aparece ya en los escritos del filósofo prusiano Immanuel Kant (1724-1804) y los geógrafos alemanes Alexander Humboldt (1769-1859) y Karl Ritter (1779-1859). En Kant la región está ampliamente relacionada con el concepto de espacio, en tanto en Humboldt y en Ritter se considera la existencia de regiones naturales.

El concepto ha evolucionado en el tiempo, en medio de la interpretación que las diversas disciplinas dan del mismo. Si bien en principio hizo referencia a las características homogéneas que posee un espacio geográfico determinado, los procesos de regionalización han observado una evolución continua en términos de los criterios que los orientan, llegando a que en su comprensión se acentúe el papel del hombre, el cual toma el rol de "modelador y conformador de la región", con lo que "Haces o flujos de relaciones, de carácter social y económico, pasan a un primer plano de consideración" (Espejo, 2003, p. 71).

El trabajo de Mateo-Rodríguez \& Bollo-Manent (2016) describe la evolución del concepto de región a partir de las reflexiones propuestas por las diferentes corrientes geográficas. En su recorrido evidencian que no existe una unidad conceptual que favorezca la comprensión del término; sin embargo, la destacan como una categoría que permite dar cuenta de las diferencias espaciales y la especificidad de los lugares, cuya definición más general alude a un espacio físico que posee características similares asociadas a identidad compartida, rasgos ambientales, sociales, culturales, históricos y/o económicos análogos².

El término región describe además fenómenos espaciales en diversas escalas de orden supranacional y subnacional. En ese sentido Soja (2014) hace referencia a escalas regionales entre lo global y lo local, con lo cual una región puede ser definida a nivel supranacional (varios países) o corresponder a una parte de un país. De manera más convencional fija el techo de este espectro en lo nacional y establece el piso en lo local.

Se observan distintas experiencias de regionalización en el mundo, entendida esta como la clasificación de los espacios geográficos con base en criterios de diferenciación previamente definidos. Una regionalización que resulta importante para la descripción, el análisis y la comprensión de los fenómenos socioeconómicos que se producen en el espacio, al tiempo que para la formulación de políticas de desarrollo, en tanto "Es indudable que los fenómenos humanos, sociales, culturales, económicos, se ordenan en el espacio geográfico, dejan sobre él sus huellas, y guardan íntima relación con la ordenación física y bilógica que sobre ese mismo espacio se produce" (Fornaguera \& Guhl, 1969, p. 11).

Los primeros ejercicios de regionalización tuvieron lugar en la escala supranacional y se presentaron con fuerza durante la posguerra, con el firme propósito de consolidar los procesos hegemónicos de las potencias mundiales encabezadas por Estados Unidos y la otrora Unión Soviética (Rodríguez Suárez, 2016). En América latina la cuestión regional y los procesos de regionalización han estado presentes; en Colombia ella tuvo sus inicios en la segunda mitad del siglo XX, pero lo regional como aspecto del diseño institucional del Estado es algo que solo tiene lugar con la Constitución de 1991 (Barrios-Suvelza, 2017).

Entre los diferentes ejercicios de regionalización para Colombia, destaca la propuesta pionera de Fornaguera \& Guhl (1969). En la misma se adopta como criterio "el epicentrismo urbano", que considera que la especialización funcional urbana crea un sistema de dependencias reciprocas con estructuras variadas y manifiestas en una red más o menos compleja, en la que el núcleo urbano principal es el epicentro en tanto juega un rol de centro económico, de servicios, de comunicación, de intercambio de información, de creación y difusión de innovaciones e ideas, para su población y área circundante.

\footnotetext{
${ }^{2}$ Otros trabajos (Czerny, 2008; Figueras et al. 2009; Tomadoni, 2016; Vázquez \& Propín, 2001) concluyen en igual sentido.
} 
De esa manera, para la regionalización se definió el área de influencia de los principales núcleos urbanos ignorando las fronteras departamentales, y la región así definida tomó el nombre de su epicentro predominante con excepción de la región caldense (en el Eje cafetero) que se consideró bicéfala (con centros en Manizales y Pereira). Desde esa perspectiva funcional se definieron las regiones de Bogotá, Barranquilla, Medellín, Cali, Bucaramanga y Caldense, que en conjunto abarcaban algo menos del 50\% del territorio nacional pero abrigaban más del $98 \%$ de su población.

Por otra parte, Fals-Borda (1996), en la tarea de favorecer la autonomía regional, el control ciudadano del poder y una amplia inclusión y participación popular en las decisiones, definió ocho regiones desde una perspectiva sociodemográfica basada en la historia y la cultura locales. Estas fueron Región Caribe, Región Pacífico Norte, Región Andina Norte, Región Andina Central, Región Andina Sur, Región Pacífico Sur, Región Orinoquia y Región Amazonia.

Molina \& Moreno (2001) elaboraron una propuesta de regionalización nodal que sirviera a la identificación de los subsistemas urbanos existentes en Colombia, para lo cual jerarquizaron los centros urbanos según su funcionalidad e identificaron las ciudades que integraban cada subsistema nodal. Desde esa perspectiva se definieron seis niveles de centro nodal según el alcance funcional, que permitieron la clasificación del país en una metrópoli nacional, 4 metrópolis subnacionales, 12 centros regionales principales, 15 centros regionales intermedios, 18 centros subregionales mayores y 55 centros subregionales intermedios.

Desde las instancias de gobierno, el DNP avanzó en diversos ejercicios de regionalización que sirvieron de base para la conformación de las regiones CORPES, que dividieron el país en cinco áreas (Costa Atlántica, Centro-Oriente, Occidente, Orinoquia, y Amazonia) para efectos de planificación y desarrollo. Se destacan el modelo de regionalización de 1969, que con fundamento en la teoría de los polos de desarrollo buscaba jerarquizar los centros urbanos desde los que se difundiría la acción de la política pública; el modelo de regionalización nodal de 1976, que pretendía configurar una red urbana con capacidad de servir de canal para la difusión del desarrollo; y la propuesta en 1976 de regiones para la descentralización administrativa, que articulaba departamentos con criterios de contigüidad geográfica y similitudes físicas, económicas y sociales (Chamorro, 1997).

Los CORPES constituyen el referente más inmediato de las RAP, en el sentido de su configuración a partir de departamentos con continuidad geográfica y con propósitos de desarrollo regional, inversión conjunta y competitividad. No obstante, a diferencia de los CORPES, que fueron definidos desde el gobierno central y dotados con una estructura administrativa y rentas básicas para inversión, las RAP se constituyen como resultado del entendimiento y acuerdo de las dirigencias (Gobernaciones y Asambleas) de las entidades territoriales departamentales comprometidas.

El artículo 306 de la Constitución (Colombia, 1991) abrió la puerta a la conformación de las Regiones Administrativas de Planificación (RAP), integradas por dos o más departamentos y cuentan "[...] con personería jurídica, autonomía y patrimonio propio. Su objeto principal será el desarrollo económico y social del respectivo territorio". El artículo 307 de la Constitución plantea además el paso de las RAP a las RET (región como entidad territorial), lo cual les brinda un grado de autonomía mucho más amplio a aquellas que hayan funcionado por lo menos cinco años, tengan un concepto favorable de la Comisión de Ordenamiento Territorial (COT) del Congreso de la República y cuenten con la aprobación en referéndum de los ciudadanos de los departamentos interesados.

La Ley Orgánica de Ordenamiento Territorial (LOOT) regula la conformación de las RAP. Ellas deben crear un Consejo Regional Administrativo y de Planificación, que funge como instancia técnica y asesora, y para el cumplimiento de los objetivos de desarrollo cuentan con un Fondo de Desarrollo Regional que financia los proyectos de inversión que respondan a las necesidades y realidades observadas. Por otra parte, dentro de las RAP se pueden conformar las Zonas de Inversión Especial, con miras a focalizar recursos destinados a romper los círculos de pobreza de lugares específicos y disminuir la disparidad existente. 
En Colombia se han conformado cuatro RAP (Figura 1), la primera de ellas fue la RAP-E ${ }^{3}$. Región Central, integrada por los departamentos de Boyacá, Cundinamarca, Meta, Tolima y Bogotá, departamentos que acogen el $29 \%$ de la población del país. Las RAP Pacífico y RAP Caribe firmaron sus convenios durante el 2017 con el objetivo de promover el desarrollo de los departamentos vinculados, a través de la consolidación de proyectos estratégicos para la competitividad de la región. Finalmente, la RAP Eje Cafetero integró los departamentos de Caldas, Quindío y Risaralda, cuya posición geográfica y cercanía espacial, cultural y productiva permitió generar en ellos ejes de desarrollo en torno a la producción y comercialización de café (Valencia et al., 2013).

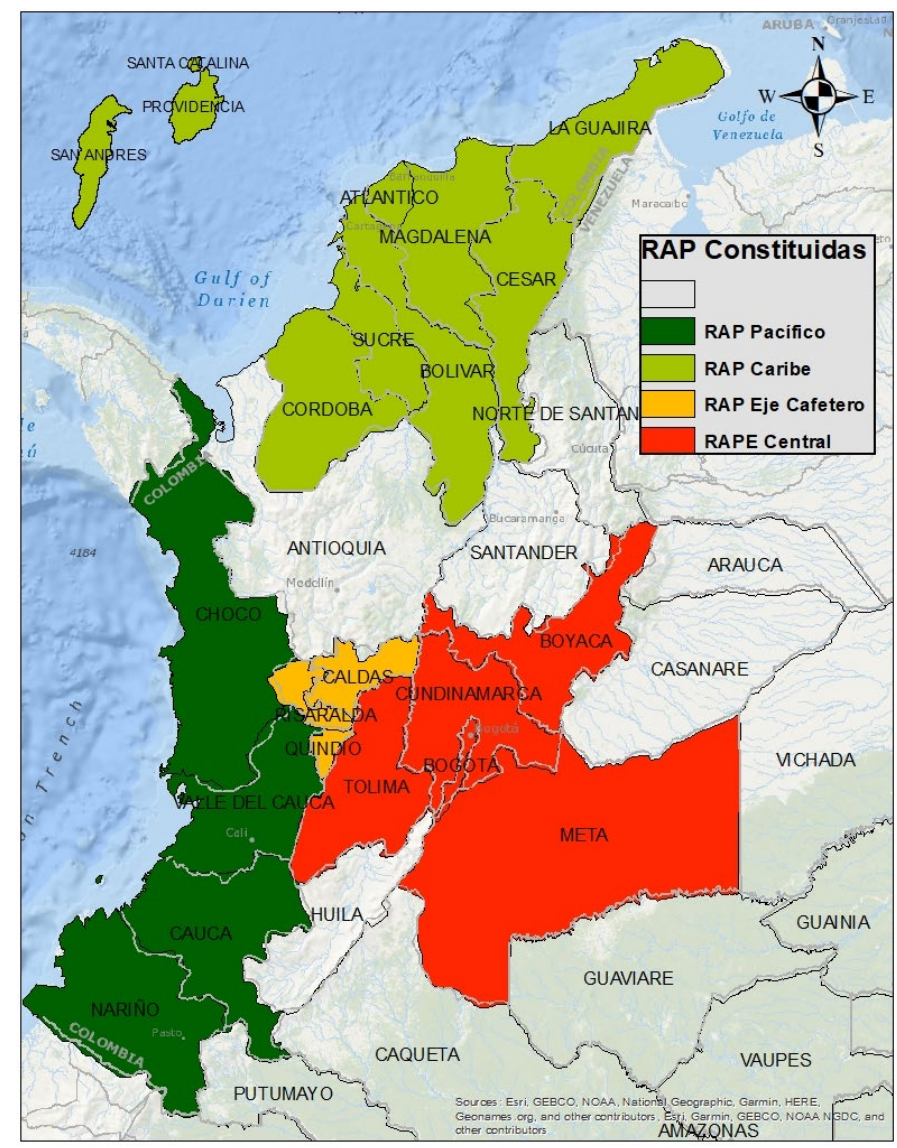

Figura 1 - Colombia, Las regiones RAP. Fuente: Elaboración propia.

El Eje cafetero es un espacio geográfico que tradicionalmente se refiere conteniendo estos tres departamentos del centro-occidente colombiano, que en conjunto constituyeron hasta 1966 uno solo, el Gran Caldas. Con ese nombre se evoca una actividad agrícola de gran significado en la historia económica, social y cultural de estos departamentos, a lo que se suma el hecho de que en un momento importante de esa historia la región fue el corazón de la actividad cafetera de Colombia.

Su primer poblamiento se dio durante el período colonial, pero sólo hasta principios del siglo XIX se inició la ocupación de este territorio, en un contexto particular de colonización, redistribución del espacio y reorganización productiva de un país, que derivó en un progresivo establecimiento de la caficultura como actividad productiva central y de exportación. Por las características de la fuerza impulsora y las facilidades de acceso a los mercados externos, el poblamiento fue más intenso en la vertiente occidental de la cordillera central, en razón a la cercanía al puerto marítimo de Buenaventura en el Pacífico colombiano; ello cambió de manera radical los patrones de asentamiento y configuró un espacio de

\footnotetext{
${ }^{3}$ RAP-E: Región Administrativa de Planificación Especial, de la que hace parte el Distrito Capital Bogotá.
} 
jerarquías urbanas con Manizales, Armenia y Pereira como las ciudades primadas de dicha vertiente (Zambrano-Pantoja \& Bernard, 1993; Arias \& Bolívar, 2006).

La economía campesina que cimentó la actividad cafetera de la zona propició la conformación de una red de beneficio, comercio y transporte del grano, que marcó el inicio de un sistema urbano con eje en las ciudades primadas e impulsó el mercado interno y la expansión industrial en el siglo XX. El desarrollo del sistema vial y de transporte, que inició en la segunda década del siglo XX en un proceso rápido e intenso y significó el salto de la mula y la arriería al cable aéreo, el ferrocarril y la carretera (García, 1978)4, fue fundamental en esa articulación de las ciudades.

Como lo anota Sanabria $(2016,2017)$, el espacio es en parte una construcción económica, política, social, cultural, y la región es de alguna manera el resultado de la forma como el sistema económico se relaciona con su espacio geográfico. En esa línea, la RAP Eje cafetero se muestra como parte de un proceso histórico marcado por la colonización y la cultura derivada del cultivo y beneficio del café; actividad que favoreció formas de relacionamiento espacial e impulsó el desarrollo de infraestructura vial propicia a la integración regional, y a ella se le asigna un papel central en la construcción y representación de la identidad colectiva de la población del Eje cafetero (Arias \& Bolívar, 2006).

El entorno cultural y el paisaje productivo vivo que se construye asociado a la actividad cafetera, fue reconocido en 2011 por la Organización de las Naciones Unidas para la Educación, la Ciencia y la Cultura (UNESCO), al declarar patrimonio cultural de la humanidad el Paisaje Cultural Cafetero de Colombia (PCC), del cual hacen parte 38 de los 53 municipios que conforman la RAP Eje cafetero.

En un trabajo reciente (Gaviria Ríos, 2020) se evidenció la abundancia de narraciones que acuden al café y la colonización como símbolos de definición del nosotros, atribuyendo al cultivo una importancia sustancial para entender los repertorios culturales que marcan el destino de la región. Al referir otros aspectos que identifican la región y despiertan apego, los actores sociales subrayan en forma repetida los elementos naturales, aquellos que los hacen sentir disfrutando de un mismo paisaje, no importa que estén en espacios de Caldas, Risaralda o Quindío. El Eje cafetero se erige como el referente socioespacial primario de pertenencia, a partir del cual se percibe la existencia de un "nosotros" y para el que se comparte una imagen de futuro en la que destacan como elementos centrales la preservación de la riqueza natural, el verde y la cultura cafetera.

Un territorio se estructura y organiza a partir de las relaciones que establecen los seres humanos, entre ellos y con el espacio ocupado y apropiado. La RAP Eje cafetero observa características que posibilitan ver en ella un territorio que avanza en su construcción. Esta región, repetida en el imaginario colectivo de su población como noción primaria de lo que fuera el Gran Caldas, sigue viva en la evidencia de los mapas producto de las elaboraciones mentales de sus habitantes, que de manera sostenida acuden a ella como espacio de referencia tradicional.

El Eje cafetero es evocado por sus habitantes como una región histórica cuyos rasgos paisajísticos, culturales y económicos característicos actúan como repertorios comunes de orgullo. Este nombre delata una condición necesaria, el café como marco cultural de una construcción social y espacial. De esa manera, la decisión de conformar la RAP Eje cafetero responde a las territorialidades presentes en ella, por lo que se augura su consolidación en el tiempo fundada en un proyecto de vida común.

\section{Análisis funcional de la RAP EJE cafetero}

Desde la geografía cuantitativa el estudio de las formas espaciales ha considerado los enfoques morfológico y funcional; el primero, asociado a una visión estática y de forma, centra su mirada en la distribución de la población sobre el territorio; el segundo, dada una comprensión del espacio a partir de

\footnotetext{
${ }^{4}$ Históricamente el sistema vial se desarrolló en tres etapas. La primera, la de los ríos y los caminos de herradura; la segunda, entre 1915 y 1930, con un desarrollo de los cables y el ferrocarril que conformaron una red complementaria; la tercera etapa, que se intensifica a partir de 1932, estuvo marcada por la aparición de las carreteras troncales, transversales e intermunicipales, que constituyeron una competencia importante al ferrocarril y tendieron a sustituir los cables aéreos.
} 
nodos y flujos, se encamina a revisar las relaciones interurbanas y en él los conceptos predominantes son los de sistema y estructura (Boix \& Trullén, 2011).

La perspectiva morfológica demanda describir la distribución de la población entre los municipios y su evolución durante el período de análisis. Esa mirada ofrece un panorama en el que, en general, los municipios de la RAP conservan su posición en el ordenamiento por tamaño poblacional, aunque se observan saltos importantes en relación con el intervalo poblacional en el que se inscriben (Tabla 1), agrupamiento en el que las capitales Pereira y Manizales amplían la brecha poblacional con su similar Armenia, Dosquebradas se perfila como subcentro regional, y Villamaría y La Tebaida observan los cambios posicionales más destacados, el primero al duplicar su población y el segundo al aumentarla en un $65 \%$ en el período de observación.

Tabla 1 - RAP Eje cafetero, población de los municipios según intervalos, 1985-2018

\begin{tabular}{|c|c|c|c|c|}
\hline \multirow{2}{*}{$\begin{array}{l}\text { INTERVALO DE } \\
\text { POBLACIÓN }\end{array}$} & MUNICIPIO & POBLACIÓN & MUNICIPIO & POBLACIÓN \\
\hline & \multicolumn{2}{|c|}{1985} & \multicolumn{2}{|c|}{2018} \\
\hline Más de 300.000 & Pereira & 303.843 & $\begin{array}{c}\text { Pereira } \\
\text { Manizales }\end{array}$ & $\begin{array}{l}467.269 \\
434.403\end{array}$ \\
\hline \multirow[t]{2}{*}{$200.001-300.000$} & Manizales & 299.414 & Armenia & 295.208 \\
\hline & Armenia & 209.870 & Dosquebradas & 217.178 \\
\hline $100001-200000$ & Dosquebradas & 101.750 & & \\
\hline \multirow[t]{6}{*}{$50.001-100.000$} & $\begin{array}{l}\text { Santa Rosa de } \\
\text { Cabal }\end{array}$ & 64.445 & $\begin{array}{l}\text { Santa Rosa de } \\
\text { Cabal }\end{array}$ & 77.838 \\
\hline & La Dorada & 56.172 & Calarcá & 72.783 \\
\hline & Calarcá & 54.216 & La Dorada & 71.905 \\
\hline & & & Villamaría & 64.652 \\
\hline & & & Chinchiná & 51.271 \\
\hline & & & Riosucio & 50.775 \\
\hline \multirow[t]{8}{*}{$30.001-50.000$} & Chinchiná & 48.125 & Montenegro & 36.751 \\
\hline & Riosucio & 44.678 & & \\
\hline & Anserma & 36.084 & Anserma & 36.149 \\
\hline & Samaná & 35.911 & & \\
\hline & Quinchía & 31.031 & La Tebaida & 33.451 \\
\hline & Montenegro & 30.653 & & \\
\hline & Quimbaya & 30.531 & Quimbaya & 30.751 \\
\hline & Villamaría & 30.219 & & \\
\hline $\begin{array}{l}\text { Menos de } 30.000 \\
\text { Población Total RAP }\end{array}$ & \multicolumn{2}{|c|}{$\begin{array}{c}38 \text { municipios } \\
1 ' 944.650\end{array}$} & \multicolumn{2}{|c|}{$\begin{array}{l}39 \text { municipios } \\
\mathbf{2} \mathbf{4} 81.560\end{array}$} \\
\hline
\end{tabular}

Fuente: Departamento Administrativo Nacional de Estadísticas (2019). 


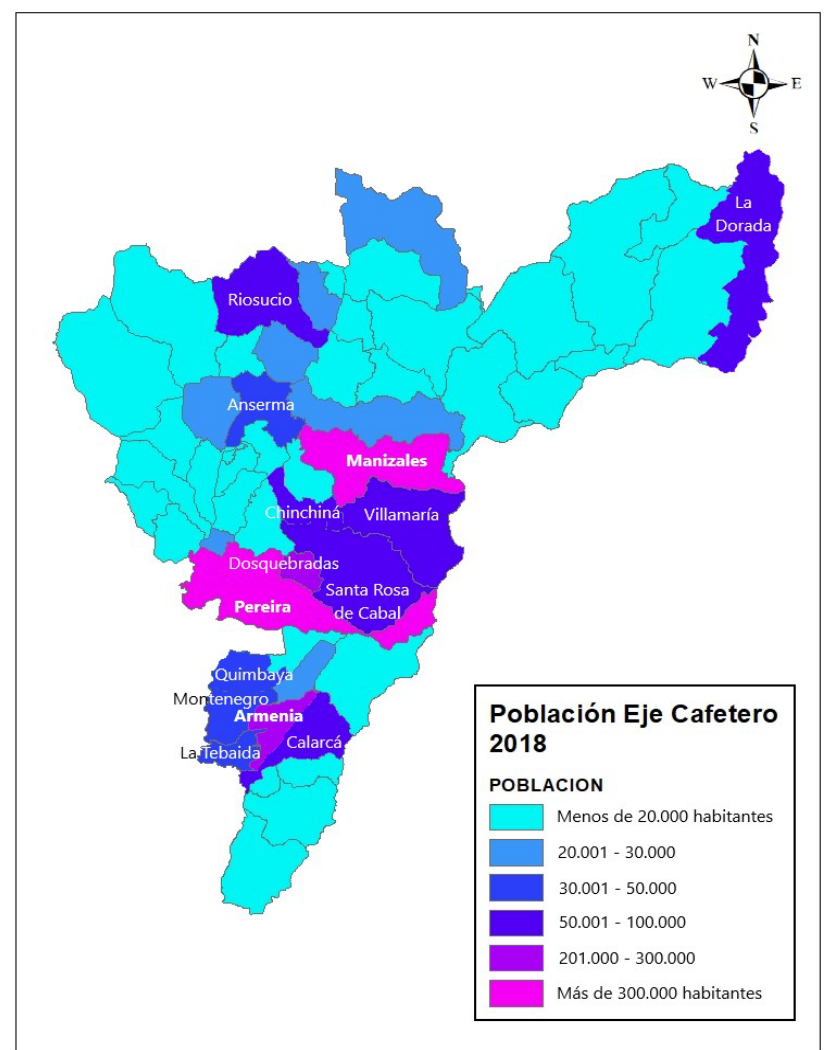

Figura 2 - RAP Eje cafetero, distribución de la población según intervalos, 2018. Fuente: Departamento Administrativo Nacional de Estadísticas (2019).

Resalta en igual sentido la disminución de población que experimentan algunos municipios, como Samaná y Quinchía, y el casi nulo crecimiento en otros, como Anserma y Quimbaya, entre los censos de 1985 y 2018. Un fenómeno en gran medida relacionado con el debilitamiento de la actividad cafetera a partir del rompimiento en 1989 del pacto de precios firmado por la Organización internacional del café (OIC), que motivó una fuerte emigración interna, hacía a las conurbaciones Pereira-Dosquebradas, Manizales-Villamaría y Armenia-Calarcá, y externa, especialmente hacia países de Europa.

De esa manera, teniendo como referencia única la distribución espacial de la población, el sistema urbano regional cuenta con dos centros regionales, Pereira y Manizales, y dos centros subregionales en Armenia y Dosquebradas (Figura 2); sistema en el que Pereira se conserva como la ciudad mayor al albergar el $18.8 \%$ de la población total de la RAP (15.6\% en 1985), seguida de cerca de Manizales que acoge el 17.5\% (15.4\% en 1985), y un poco más atrás Armenia que concentra el 11.9\% (10.8\% en 1985).

Los trabajos de Zambrano-Pantoja \& Bernard (1993), Cuervo \& González (1997) y Pachón (2013), que develan el proceso histórico de conformación del sistema de ciudades en el Eje cafetero, destacan la consolidación de Manizales, Armenia y Pereira como ciudades principales de la vertiente occidental de la cordillera central, a través de un proceso asociado a las dinámicas de expansión industrial, la producción cafetera y el desarrollo de infraestructura vial; ciudades a partir de las cuales se ha fortalecido una red urbana, aprovechando la proximidad espacial y las cercanías históricas y económicas. Ello sugiere revisar otros enfoques para identificar los centros de una región con raíces históricas y económicas, como la RAP Eje cafetero.

En una perspectiva más dinámica, algunas técnicas de identificación consideran a dichos centros como las unidades de mayor orden en un sistema urbano, caracterizadas por proveer los bienes y servicios de mayor nivel y realizar las funciones más centrales en la jerarquía urbana; en tanto otras recurren al análisis de los flujos de interacción existentes entre las unidades urbanas que integran la formación, estimando a partir de ello índices de centralidad que permiten jerarquizar estas unidades (Boix \& Trullén, 2011) ${ }^{5}$.

\footnotetext{
${ }^{5}$ En Gaviria Ríos (2018) se desarrolla un ejercicio de este tipo aplicado a la Ciudad región Eje cafetero.
} 
El enfoque funcional tiene su origen en la Teoría del lugar central (TLC). La idea transmitida por la TLC destaca que los centros son a grandes rasgos sinónimos de las ciudades que se constituyen en ejes de los sistemas regionales al actuar como polos financieros, de negocios y de empleo; a la vez son fuente principal en el suministro de bienes y servicios de mayor complejidad, como tratamiento hospitalario, educación superior y entretenimiento. Por tanto, el concepto general de lugar central hace referencia a una aglomeración urbana que es eje de una región, una condición de centralidad que es definida por su función comercial y de servicios con respecto a su hinterland.

Los lugares centrales varían en importancia de acuerdo con las funciones que cumplen, con lo que los centros de orden superior son aquellos que ofertan una amplia variedad de mercancías y servicios, en tanto los centros de orden inferior disponen de una variedad más limitada de estas funcionalidades, es decir, alguna parte de la gama ofrecida por el centro superior. En términos instrumentales, la teoría del lugar central definió la centralidad de un núcleo urbano como la razón entre todos los servicios allí proporcionados (tanto para sus residentes como para los visitantes procedentes de su región complementaria) y los servicios necesarios solo para dichos residentes. Las ciudades con una alta centralidad proporcionan muchos servicios por residente, al contrario de las de baja centralidad.

De esa manera se puede establecer una jerarquía de lugares centrales de acuerdo con las funciones económicas, comerciales y de servicios que ellos prestan a la región complementaria, con lo que en esta perspectiva tamaño e importancia son dos conceptos diferentes; un municipio o ciudad pequeña puede desempeñar funciones de un orden superior a las de un municipio de mayor dimensión poblacional. La función de centralidad de una ciudad viene dada por la concentración de servicios y bienes que ella proporciona y su importancia depende de ese grado de funcionalidad.

Partiendo de esos principios, la identificación de los centros y subcentros regionales de la RAP Eje Cafetero puede partir de la caracterización funcional de los municipios. En la línea propuesta, en Colombia se cuenta con trabajos como el de Molina \& Moreno (2001), que avanzaron en la definición de una regionalización nodal del país y aportaron información que permite identificar los centros urbanos de orden nacional y regional a partir de criterios de funcionalidad.

En la clasificación de Molina y Moreno, 11 municipios de la RAP Eje cafetero quedaron incluidos entre el tercer y sexto orden jerárquico establecido; Pereira, Manizales y Armenia, fueron categorizadas como centros regionales principales; Santa Rosa de Cabal, fue considerado un centro subregional mayor; Dosquebradas, La Dorada, Anserma, Belén de Umbría, Manzanares, Santuario y Chinchiná, fueron caracterizados como centros subregionales intermedios. Los demás municipios estaban equipados con funciones urbanas de alcance local, por lo que no llegaron a ser incluidos en alguna de las categorías establecidas para el modelo de ordenamiento.

Un caso particular es el de Dosquebradas, el cuarto municipio en población de la región pero que según esa clasificación desempeña un papel subregional intermedio con una jerarquía de tercer nivel poco congruente con su talla poblacional; algo que evidencia su condición de ciudad conurbada a la capital (Pereira), que ha crecido como parte de una dinámica de polarización social en la ocupación de los núcleos urbanos, fenómeno que es definido en Molina \& Moreno (2001) como hiperurbanización metropolitana sin desconcentración funcional.

En el estudio sobre la Ecorregión Eje Cafetero (Corporación Alma Mater, 2002) se realizó un trabajo complementario de jerarquización funcional de los centros urbanos, utilizando información sobre la presencia o no de funciones relevantes para las actividades económicas, institucionales, sociales y de servicios. Los municipios de la ecorregión fueron ordenados de mayor a menor en razón al número de funciones ofrecidas entre 70 consideradas, las que a su vez se ordenaron en relación directa con la intensidad de su presencia en los diferentes centros urbanos. De esta manera se categorizaron los municipios de acuerdo con la cantidad y la especialización de sus funciones.

Con un resultado similar, las capitales Manizales, Pereira y Armenia evidenciaron un orden funcional de primer nivel al asumir el rol de centros regionales principales, con influencia a escala regional y subregional. Por otra parte La Dorada, un municipio localizado al extremo nororiental de la RAP, fue clasificado en un segundo nivel como centro subregional mayor por contar con funciones relevantes para el nororiente del departamento de Caldas; en tanto Calarcá, que cumplió con el rango de funciones para esta categoría, fue subordinado a centro subregional intermedio, en condición similar a otros como Riosucio y Chinchiná, por no contar con amplia infraestructura de apoyo a las actividades económicas, como centrales de abasto, centros de procesamiento cárnico (frigoríficos), centros de transformación 
agrícola y centros de procesamiento industrial de derivados del café. El resto de municipios observó jerarquías inferiores de centro urbano o centro rural, en tanto su funcionalidad era de orden local.

La Tabla 2 resume los resultados de un ejercicio de actualización del escalograma de funciones de los municipios de la formación espacial, para lo cual se tuvo como referente el listado utilizado en el trabajo sobre la Ecorregión Eje Cafetero y se hizo uso de los datos sobre oferta de funciones obtenidos mediante consulta a las oficinas de planeación de los municipios. A partir de esa información se destacan nuevos hechos que resultan significativos para la jerarquización funcional del sistema de ciudades de la RAP Eje cafetero.

Tabla 2 - RAP Eje cafetero, orden funcional de las unidades urbanas, 2018

\begin{tabular}{ccc}
\hline Orden funcional & $\begin{array}{c}\text { Rango de } \\
\text { funciones }\end{array}$ & Municipios de la formación espacial \\
\hline $\begin{array}{c}\text { Centros Regionales Principales } \\
\text { Centros Subregionales Mayores } \\
\text { Centros Subregionales Intermedios }\end{array}$ & $\begin{array}{c}58 \text { o más } \\
\text { De } 50 \text { a } 57 \\
\text { De } 43 \text { a } 49\end{array}$ & $\begin{array}{c}\text { Santa Rosa de Cabal, Riosucio, Salamina, Calarcá, Anserma, Supía, } \\
\text { Neira, Villamaría }\end{array}$ \\
Centros Urbanos & De 36 a 42 & $\begin{array}{c}\text { Montenegro, San José, Manzanares, Risaralda, Aguadas, Palestina, } \\
\text { Quimbaya, Chinchiná, Marquetalia, La Tebaida, Marulanda, } \\
\text { Dosquebradas, Marmato, Pensilvania }\end{array}$ \\
$\begin{array}{c}\text { Centros Rurales de Servicios } \\
\text { Centros rurales }\end{array}$ & De 30 a 35 & $\begin{array}{c}\text { Apía, Salento, Filadelfia, Circasia, Filandia, Guática, Belén de Umbria } \\
\text { Victoria, Pijao, Pácora, Marsella, Belalcazar, Pueblo Rico, Norcasia, } \\
\text { Génova, Ballboa, Buenavista, Córdoba, Aranzazu, Santuario, La } \\
\text { Virginia, La Merced, Quinchia, Mistrató, La Celia, Samaná, Viterbo. }\end{array}$ \\
\hline Menos de 30
\end{tabular}

Fuente: Elaboración con base en información suministrada por las oficinas de planeación de los municipios de la Ciudad región Eje Cafetero, a noviembre de 2018 y la clasificación propuesta en Corporación Alma Mater (2002).

De un lado, las tres ciudades capitales conservan su carácter de centros principales a nivel regional, condición que igualmente alcanza el municipio de La Dorada. Al contrario, los municipios conurbados a las capitales, como Dosquebradas, Villamaría y Calarcá observan una baja funcionalidad que solo permite clasificarlos como centros urbanos, el primero, o centros subregionales intermedios, los demás. En esto se evidencia una configuración funcional cercana a lo propuesto por la Teoría del Lugar Central, que basada en el supuesto de superficies isotrópicas considera que los asentamientos con órdenes de funcionalidad inferior se distribuyen de manera más uniforme en el espacio regional y con mayor proximidad a los centros principales; por lo que estos municipios se muestran altamente dependientes de los servicios y funciones de mediana y alta complejidad ofrecidos por su ciudad capital. Al contrario, y cercano a esa idea normativa, La Dorada, un municipio más distante de las principales aglomeraciones urbanas de la región, observa una centralidad funcional ampliamente superior.

De otro lado, en relación con los hallazgos del estudio de la Ecorregión (Corporación Alma Mater, 2002), se observan cambios en la centralidad de los municipios de la región; entre ellos se desatacan los casos Santa Rosa de Cabal, Salamina, Anserma, Supía, Neira y Villamaría, que fortalecen su funcionalidad y alcanzan a compartir el nivel de centros subregionales intermedios con Riosucio y Calarcá; una situación contraria a la de Chinchiná, que pierde su condición de centro subregional intermedio y pasa a ser centro urbano, y La Virginia, que observa un fuerte retroceso en su jerarquía funcional en el que pierde su condición de centro urbano y adquiere la de centro rural, en un proceso de centralización y dependencia institucional respecto a la capital departamental, Pereira.

Una perspectiva que resulta más integral para calificar la jerarquía funcional de los municipios es el índice ponderado de centralidad. Este Índice permite medir la complejidad funcional en términos no solo del número de funciones en un sitio, sino también de su frecuencia de ocurrencia. Es decir, para su cálculo, a las funciones les es asignado un peso en proporción inversa a la frecuencia con la cual ellas tienen presencia en las ciudades del sistema. El índice de centralidad para una ciudad es, entonces, la sumatoria de los pesos relativos de las funciones encontradas allí ${ }^{6}$, con lo que entre mayor el índice tanto mayor la complejidad funcional de esa ciudad (Tabla 3 ).

\footnotetext{
${ }^{6}$ Siendo Pi: peso de la función "i", Fi: frecuencia de la función "i" en el sistema, N: valor combinado de centralidad, una constante que se asume igual a 100; entonces, $P_{i}=N / F_{i}$. El índice de centralidad (C) de la ciudad “j” queda definido como $C=\sum P_{i j}$; con Pij: función i con presencia en la ciudad $\mathbf{j}$.
} 
Tabla 3 - RAP Eje cafetero, índice ponderado de centralidad de los municipios

\begin{tabular}{|c|c|c|}
\hline Municipio & Índice de centralidad (2018) & $\begin{array}{l}\text { Posición por tamaño poblacional } \\
\qquad(2018)\end{array}$ \\
\hline Pereira & 579,95 & 1 \\
\hline Armenia & 467,83 & 3 \\
\hline Manizales & 418,84 & 2 \\
\hline La Dorada & 344,49 & 6 \\
\hline Villamaría & 182,43 & 9 \\
\hline Calarcá & 180,57 & 5 \\
\hline Dosquebradas & 169,22 & 4 \\
\hline Supía & 168,80 & 20 \\
\hline Riosucio & 164,20 & 8 \\
\hline Santa Rosa de Cabal & 159,77 & 7 \\
\hline Pensilvania & 159,47 & 21 \\
\hline Anserma & 157,23 & 15 \\
\hline La Tebaida & 143,50 & 11 \\
\hline Salamina & 139,97 & 30 \\
\hline Chinchiná & 137,97 & 10 \\
\hline Quimbaya & 132,08 & 13 \\
\hline Marmato & 131,62 & 40 \\
\hline Aguadas & 127,70 & 25 \\
\hline Neira & 126,92 & 17 \\
\hline Marulanda & 117,66 & 52 \\
\hline Marquetalia & 117,10 & 32 \\
\hline Palestina & 108,80 & 27 \\
\hline Manzanares & 108,80 & 24 \\
\hline Risaralda & 101,11 & 41 \\
\hline Montenegro & 97,15 & 12 \\
\hline San José & 92,66 & 44 \\
\hline Belén de Umbría & 88,78 & 19 \\
\hline Guática & 87,64 & 31 \\
\hline Pácora & 86,33 & 36 \\
\hline Filandia & 85,65 & 34 \\
\hline Marsella & 85,45 & 23 \\
\hline Circasia & 85,31 & 18 \\
\hline Filadelfia & 82,54 & 34 \\
\hline Apía & 82,42 & 26 \\
\hline Salento & 80,24 & 46 \\
\hline Belalcázar & 78,93 & 38 \\
\hline Génova & 74,96 & 45 \\
\hline Viterbo & 74,27 & 35 \\
\hline Pijao & 71,80 & 49 \\
\hline Victoria & 69,68 & 43 \\
\hline Norcasia & 67,19 & 48 \\
\hline Córdoba & 65,87 & 50 \\
\hline Buenavista & 64,37 & 53 \\
\hline Balboa & 62,90 & 47 \\
\hline Pueblo rico & 62,48 & 33 \\
\hline La Virginia & 62,05 & 16 \\
\hline Aránzazu & 60,24 & 37 \\
\hline Santuario & 59,83 & 29 \\
\hline Quinchía & 50,36 & 14 \\
\hline La Merced & 49,78 & 51 \\
\hline Mistrató & 44,79 & 28 \\
\hline La Celia & 39,54 & 42 \\
\hline Samaná & 37,77 & 22 \\
\hline
\end{tabular}

Fuente: Elaboración propia a partir de información de las oficinas de planeación de los municipios. 
Este nuevo índice confirma que los municipios con mayor nivel de centralidad en la RAP Eje Cafetero son las ciudades capitales (Pereira, Armenia y Manizales), en tanto que el municipio de La Dorada sigue destacándose como un centro regional en el oriente del departamento de Caldas. Pereira se muestra como el centro regional con mayor índice de centralidad, seguido de Armenia y Manizales; en esa condición ha resultado determinante la prestación de servicios para su área metropolitana y el ser la única ciudad capital de la región en la que se localiza un aeropuerto internacional. Por otro lado, las diferencias de centralidad entre Manizales y Armenia están marcadas por la ausencia de servicios aeroportuarios en esta última, lo cual es ampliamente compensado por su mayor organización en el servicio de abastos de productos de la canasta familiar.

Lo que resalta al considerar la frecuencia de ocurrencia de las funciones es la mejora sustancial que se observa en la condición de centralidad de Dosquebradas, municipio que desde esta perspectiva alcanza niveles cercanos a los de sus similares conurbados Calarcá y Villamaría. En coherencia con ello, si se considera como criterio de identificación de los centros el número de unidades económicas instaladas en los municipios, Dosquebradas se caracteriza por albergar una actividad económica considerable, cercana en términos absolutos y relativos a la que se desarrolla en una capital como Armenia y superior en términos de densidad a los índices que presentan todas las ciudades capitales de la región (Gaviria Ríos, 2017). Este es un municipio con gran concentración de actividad industrial, que además cuenta con la presencia de algunas empresas de servicios comerciales, financieros y logísticos, que en conjunto constituyen una fuente de oportunidades de empleo importante. Es por lo mismo que, de acuerdo con las estimaciones de Alfonso (2010), Dosquebradas generaba algo más del 60\% de los puestos de trabajo demandados por sus residentes ocupados.

En síntesis, desde los puntos de vista morfológico y funcional es claro que las capitales Pereira y Manizales se destacan como centros regionales principales. Aunque en una perspectiva morfológica Armenia y La Dorada no alcanzan esa condición, su amplio desempeño funcional permite calificar a ambos como centros regionales principales. En sentido diferente, si bien Dosquebradas es el cuarto municipio en población y en él se concentra una actividad económica importante, el bajo número de funciones que alberga sólo permite calificarlo como centro subregional intermedio; una condición que comparte con sus similares Calarcá, Villamaría, Riosucio y Supía.

En la Figura 3 están referenciados los centros regionales y subregionales definidos a partir del criterio funcional. Estos están en amplia medida concentrados en las aglomeraciones urbanas alrededor de las ciudades capitales; no obstante, en el departamento de Caldas se evidencia una mejor distribución espacial de estas jerarquías urbanas, condición que beneficia las poblaciones de las zonas noroccidental y nororiental de la región. En todo caso, como se visualiza en la figura, la distribución de los lugares centrales de primero y segundo orden en la RAP Eje cafetero favorece la accesibilidad de todos los habitantes para la atención a sus necesidades diversas. 


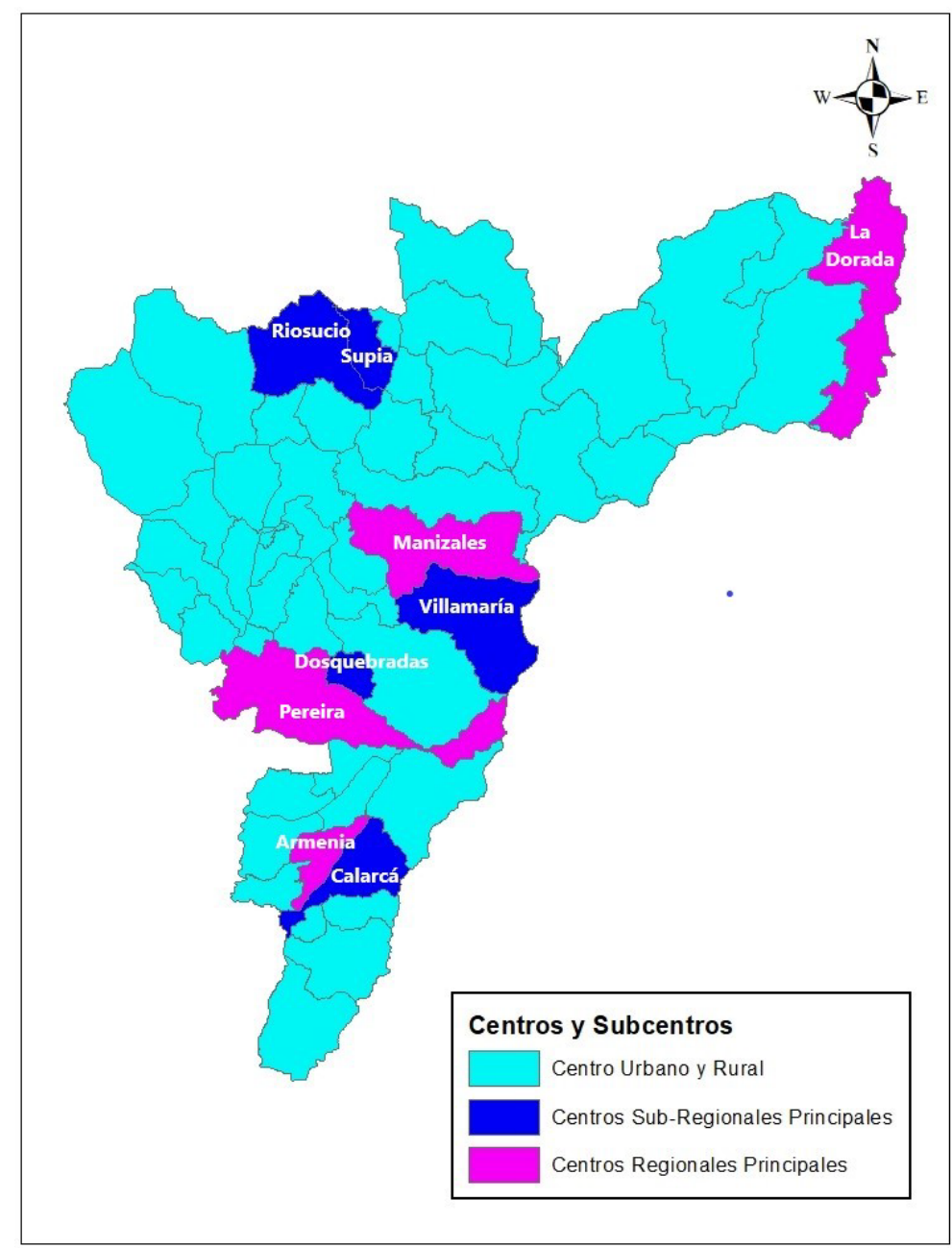

Figura 3 - RAP Eje cafetero, centros y subcentros regionales, perspectiva funcional. Fuente: Elaboración propia.

\section{Conclusiones}

Las nuevas realidades geográficas derivadas del proceso de globalización exigen figuras territoriales flexibles que se puedan conformar fácilmente con base en la voluntad de los gobiernos territoriales, que impulsen estrategias de desarrollo eficaces y construyan o reconstruyan procesos regionales sólidos a partir de los municipios y departamentos. En este contexto, los esquemas asociativos de entidades territoriales promovidos, entre otras, por la Ley Orgánica de Ordenamiento Territorial (LOOT) adquieren una relevancia particular.

Las figuras asociativas son instrumentos de articulación y desarrollo regional, que parten de las propias entidades territoriales para abordar problemas y temáticas conjuntas. Ellas se consolidan en el tiempo en la medida que se fundamenten en una clara identidad territorial, lo cual guarda relación con la dimensión espacial que desarrolla la población, la pertenencia y los valores que atribuye al espacio habitado, es decir, con su grado de configuración como territorio.

La RAP Eje cafetero observa características que posibilitan ver en ella un territorio que avanza en su construcción. Esta región se erige como referente primario de pertenencia para su población. De esa manera, la decisión de conformarla responde a las territorialidades presentes en ella, por lo que se augura su consolidación en el tiempo fundada en un proyecto de vida común.

A través de un ejercicio de caracterización funcional de la RAP Eje Cafetero se pudo observar que dicha formación espacial presenta una organización de carácter policéntrico, en la que las tres ciudades capitales aparecen como centros principales con capacidad de alternarse el rol de liderazgo en el impulso 
al desarrollo de la región, en tanto que el municipio de La Dorada se configura como un centro urbano relevante en la naciente región y funge como un polo de desarrollo para su zona nororiental.

En sus orígenes, estos nodos centrales se desplegaron a partir de un proceso paralelo de expansión industrial y de producción cafetera, que explica la conformación de un eje urbano industrial en la zona cafetera, en el cual los accidentes geográficos, como la topografía montañosa característica, determinaron la existencia de reducidas áreas de influencia de cada nodo y favorecieron la diversificación del sistema urbano en formación. La crisis del café en los años noventa del siglo XX, que significó la pérdida de más de 60.000 empleos y un retroceso de dos puntos porcentuales en la contribución regional al producto nacional, obligó a las entidades territoriales de la región a repensar sus economías como complemento a las acciones orientadas a la renovación la actividad cafetera, mediante la diferenciación del producto y la incorporación de mayor valor agregado. Pereira refrescó su centralidad a partir del desarrollo comercial y financiero, Manizales hizo lo propio a través de un ambicioso programa de transformación de ciudad universitaria a eje del conocimiento, y Armenia le apostó a los proyectos agroindustriales y a ser destino para las convenciones, esto último en articulación con el agro y ecoturismo que desarrollaron sus municipios aledaños.

La condición de centro principal de La Dorada tiene relación con su localización estratégica, que le ofrece ventajas para la conectividad con la costa norte, por medio del rio Magdalena y la Ruta del Sol, y con el centro del país. Esa localización favorece además su articulación a los desarrollos del oriente de Caldas, norte del Tolima, suroccidente de Santander, noroccidente de Cundinamarca, suroriente de Antioquia y occidente de Boyacá. Es un centro que, en todo caso, se configura aislado de sus similares en la región con fundamento en sus potencialidades y atiende una población que por distancia está fuera del área de influencia del resto de centros regionales.

Las ventajas de ese policentrismo intra-regional están en discusión. Algunas entidades como la Unión Europea consideran en su estrategia territorial que el policentrismo es un ordenamiento espacial que favorece la disminución de los desequilibrios territoriales y un desarrollo orientado hacia una mayor cohesión territorial, entendida esta última como un principio orientador de una política pública encaminada al logro de propósitos como la articulación de las comunidades (cohesión social), el acceso equitativo a servicios y equipamiento (equidad espacial) y construcción de un proyecto regional común (identidad territorial) partiendo del respeto a la diversidad y las características propias (Fernández-Tabares et al., 2009).

En una idea opuesta, el policentrismo se considera un obstáculo para el desarrollo regional en tanto es una configuración espacial en la que no existe un liderazgo definido, por lo que en ella se favorece el conflicto de intereses y la consecuente desarticulación de las entidades territoriales que la integran. Contrario a ello, una condición monocentrica asegura la presencia de una entidad que comanda el desarrollo y es fuente de externalidades positivas para el resto de la región.

La estructura del sistema urbano tradicional monocéntrico responde a un patrón definido en la teoría del lugar central, donde la ciudad central ejerce una jerarquía dominante sobre el resto de las ciudades de su entorno. La literatura reciente (Gaviria Ríos, 2018) pone de relieve que los patrones de articulación de los sistemas urbanos están evolucionando desde estructuras verticales (jerarquías) monocéntricas, hacia estructuras horizontales policéntricas, denominadas redes de ciudades.

El sistema urbano de la RAP Eje cafetero hay presencia de relaciones de red entre las ciudades, especialmente entre las que hacen parte de sus zonas de metropolización; vínculos que generan una interacción espacial de orden socioeconómico, que se fundamentan en la cercanía geográfica y cultural, y a través de los cuales se generan sinergias de distinto orden que derivan en economías externas de red (Gaviria Ríos, 2018). En ese sentido, se puede hacer referencia a un policentrismo en red como elemento de configuración espacial en la región.

Finalmente, cabe advertir que, si bien desde el enfoque de la teoría del lugar central se ha planteado como opción de análisis funcional la construcción de un escalograma de funciones para definir el orden funcional y el nivel de centralidad de las unidades que integran un sistema espacial, en otros trabajos (Escolano Utrilla et al., 2007) se ha demostrado la conveniencia de diseños alternativos para identificar la estructura y jerarquía funcional de los centros urbanos. Estos diseños tienen como fundamento la especialización productiva, 
entendida como el inverso de la diversidad funcional y calculada a través de índices de Theil de distribución de la población ocupada en las distintas actividades económicas; y la intensidad de esa especialización en cada unidad urbana, calculada mediante el coeficiente de localización.

De manera complementaria, a partir del trabajo de Escolano Utrilla et al. (2015) se bosqueja una ruta interesante para la identificación de centros y subcentros urbanos en un sistema de ciudades, esto desde la perspectiva de los núcleos de empleo. Dicho ejercicio investigativo se centra en explorar el alcance de la dispersión espacial y funcional de los empleos en una región urbana, identificando y caracterizando posibles subcentros de empleo y delimitando las cuencas de atracción de los mismos. Sin embargo, para el caso en estudio, no fue posible recurrir a estos métodos alternativos de identificación de centros y subcentros regionales, dado que ello exige contar con información sobre variables de empleo en la escala municipal.

\section{Referencias}

Alfonso, 0. (2010). Impactos socioeconómicos y demográficos de la metropolización de la población colombiana y de los mercados de trabajo y residenciales (Documentos de Trabajo, No. 31). Bogotá: Universidad Externado de Colombia.

Arias, J., \& Bolívar, Í. (2006). El cultivo de la identidad natural. Paisaje, cultura y turismo en Montenegro, Quindío. In: I. Bolívar (Editora), Identidades culturales y formación del estado en Colombia: colonización, naturaleza y cultura (pp. 51-118). Bogotá: Ediciones Uniandes.

Barrios-Suvelza, F. (2017). El impacto de las recientes reformas de regiona-lización en Colombia, Ecuador, Perú y Bolivia: conceptos y procesos. Geopolítica(s): Revista de Estudios sobre Espacio y Poder, 8(1), 51-89.

Boix, R., \& Trullén, J. (2011). Policentrismo y estructuración del espacio: una revisión crítica desde la perspectiva de los programas de investigación. Architecture, City and Environment, (18), 27-54.

Chamorro, L. (1997). Balance del proceso de conformación de regiones en Colombia. Planeación \& Desarrollo, 28(4), 115-150.

Colombia. (1991, 7 de julio). Art. 306 y 307. Colombia: Constitución Política de Colombia.

Colombia. Departamento Nacional de Planeación - DNP. (2013). Definición legal y funcional de los esquemas asociativos de entidades territoriales en Colombia. Bogotá. Recuperado el 5 noviembre de 2018, de https://colaboracion.dnp.gov.co/CDT/Desarrollo\%20Territorial/DEFINICI\%C3\%93N\%20LEGAL\%20Y\%20FUNCI ONAL\%20DE\%20LOS\%20ESQUEMAS\%20ASOCIATIVOS\%20DE\%20ENTIDADES\%20TERRITORIALES\%20EN\%2 OCOLOMBIA.pdf

Corporación Alma Mater. (2002). Ecorregión Eje cafetero: un territorio de oportunidades. Pereira: Corporación Alma Mater.

Cuervo, L., \& González, J. (1997). Industria y ciudades, en la era de la mundialización: un enfoque socioespacial. Bogotá: Tercer Mundo Editores.

Czerny, M. (2008). Regiones y sociedades regionales frente a la globalizacion. Espacio y Desarrollo, 30(20), 19-30. Recuperado 10 septiembre de 2018, de http://ezproxy.net.ucf.edu/login?url=http://search.ebscohost.com/login.aspx?direct=true \&db=aph \&AN=443867 $71 \&$ site $=$ ehost-live

Departamento Administrativo Nacional de Estadísticas (2019). Demografía y población [Base de datos]. Recuperado el 15 noviembre 2019, de https://www.dane.gov.co/index.php/estadisticas-por-tema/demografia-ypoblacion.

Escolano Utrilla, S., Ortiz Véliz, J., \& Moreno Mora, R. (2007). Globalización y cambios funcionales recientes en las ciudades del sistema urbano chileno. Cuadernos Geográficos, (41), 33-60. 
Escolano Utrilla, S., Ortiz Véliz, J., \& Moreno Mora, R. (2015). La estructura de los núcleos de empleo de la región metropolitana de Santiago (Chile): centralización funcional y espacial. Boletín de la Asociación de Geógrafos Españoles, (69), 263-290. http://dx.doi.org/10.21138/bage.1897.

Espejo, C. (2003). Anotaciones en torno al concepto de región. Nimbus, (11-12), 67-87. Recuperado el 17 de abril de 2018, de http://dialnet.unirioja.es/servlet/articulo?codigo=839169

Fals-Borda, O. (1996). Región e historia. Bogotá: Tercer Mundo Editores.

Fernández-Tabares, A., Pedregal-Mateos, B., Rodríguez-Mateos, J., Pita-López, M., \& Zoido-Naranjo, F. (2009). El concepto de cohesión territorial. Escalas de aplicación, sistemas de medición y políticas derivadas. Boletín de la A.G. E., (50), 157-172.

Figueras, A., Capello, M., \& Moncarz, P. (2009). Un ejercicio de agrupación territorial: ensayo académico con vistas a salvar problemas. Ensayos de Política Económica, (3), 65-84. Recuperado el 4 de mayo de 2018, de http://bibliotecadigital.uca.edu.ar/repositorio/revistas/ejercicio-agrupacion-territorial-ensayo-academico.pdf

Fornaguera, M., \& Guhl, E. (1969). Colombia: ordenación y territorio con base en el epicentrismo regional. Bogotá: Universidad Nacional de Colombia. Recuperado el 12 de febrero de 2019, de http://www.cid.unal.edu.co/files/publications/CID196901foorte.pdf

García, A. (1978). Geografía económica de Caldas. Bogotá: Banco de la República.

Gaviria Ríos, M. (2017). Configuración espacial de la formación urbana regional del Eje Cafetero colombiano. Cuadernos de Geografía: Revista Colombiana de Geografía, 26(1), 155-170. http://dx.doi.org/10.15446/rcdg.v26n1.56666.

Gaviria Ríos, M. (2018). Estructura de la red de ciudades en la ciudad región Eje cafetero (Colombia). Economía, Sociedad y Territorio, 18(58), 919-945. http://dx.doi.org/10.22136/est20181241.

Gaviria Ríos, M. A. (2020). Territorialidades en la ciudad-región Eje Cafetero, Colombia. Territorios, (42), 1-24. http://dx.doi.org/10.12804/revistas.urosario.edu.co/territorios/a.7012.

Mateo Rodríguez, J., \& Bollo-Manent, M. (2016). La región como categoría geográfica. México: Universidad Nacional Autónoma de México. http://dx.doi.org/10.22201/ciga.9786070279133e.2016.

Molina, H., \& Moreno, P. (2001). Aportes para una nueva regionalización del territorio colombiano. In , O. Alfonso (Ed.), Ciudad y región en Colombia (pp. 581-693). Bogotá: Universidad Externado de Colombia.

Pachón, Á. (2013). La infraestructura de transporte y el sistema de ciudades en Colombia durante el período 19381951. RegionEs, 8(1), 5-34.

Rodríguez Suárez, P. M. (2016). América Latina y América del Norte ante los procesos de regionalización mundial. Oasis, 24(24), 27-44. http://dx.doi.org/10.18601/16577558.n24.03.

Sanabria, S. (2016). Progreso tecnológico y asimetrías en el crecimiento económico regional en Colombia: periodo 1980-2010 (Tesis doctoral). Facultad de ciencias económicas, Universidad Nacional de Colombia, Bogotá. Recuperado el 24 de abril de 2020, de http://bdigital.unal.edu.co/55073/1/74392230.2016.pdf

Sanabria, S. (2017). Progreso tecnológico y divergencias regionales: evidencia para Colombia (1980-2010). Investigaciones Regionales Journal of Regional Research, (38), 7-25. Recuperado el 23 de abril de 2020, de https://investigacionesregionales.org/wp-content/uploads/sites/3/2017/11/01-SANABRIA.pdf

Soja, E. (2014). En busca de la justicia espacial. Valencia, España: Editorial Tirant Humanidades.

Tomadoni, C. (2016). Regionalismo estratégico: una respuesta frente a la glocalización. El desarrollo urbano posfordista en el este de Alemania. Geograficando, 12(1), 1-14. Recuperado el 2 de febrero de 2019, de http://www.memoria.fahce.unlp.edu.ar/art_revistas/pr.7427/pr.7427.pdf

Valencia, F. H., Cortázar-Gómez, D. M., \& López-Soto, A. M. (2013). Composición de la Economía de la Región Eje Cafetero de Colombia. Ensayos Sobre Economía Regional, 54, 1-54. http://dx.doi.org/10.32468/eser.54. 
Vázquez, V., \& Propín, E. (2001). Las diferencias regional-económicas del Estado de Guerrero, México. Investigaciones Geográficas, (46), 131-147.

Zambrano-Pantoja, F. y Bernard, O. (1993). Ciudad y territorio: el proceso de poblamiento en Colombia (Travaux de l'Institut Français d'études Andines, No. 64). Lima: Institut Français d'études Andines, Academia de historia de Bogotá. http://dx.doi.org/10.4000/books.ifea.2083.

Editor: Rodrigo Firmino

Recibido: Feb. 21, 2020

Aprobado: Jun. 05, 2020 